\title{
Acknowledgement of Reviewers for 2021
}

Accepted: 7 January 2022/Published online: 16 February 2022

(C) The Author(s), under exclusive licence to Springer Nature Switzerland AG 2022

The Editors-in-Chief and the Publisher wish to acknowledge the assistance of the following colleagues, scientists across the world helping us to review articles for our Journal in tight timeframes during the year of 2021. The Editors-in-Chief are very grateful for all the reviewers for providing in-depth review reports for authors to improve the quality of the scripts.

\author{
Abbas Abbaszadeh Shahri \\ Ahmed Abdelmaksoud \\ Ilmutdin Abdulagatov \\ Fidelis Abija \\ Suranto Ahmad Muraji \\ Mohammadali Ahmadi \\ Zulkifl Ahmed \\ Mohammad Ayaz Alam \\ Marwan Alheib \\ Claudio Alimonti \\ Watheq Al-Mudhafar \\ Danial Jahed Armaghani \\ Muhammad Aziz \\ Qingsheng Bai \\ Adam Bajcar \\ Sylvain Barbot \\ Andreas Benardos \\ Christof Beyer \\ Daniel Brito \\ Loes Buijze \\ Qingwang Cai \\ Shaobo Chai \\ D. Chandrasekharam \\ $\mathrm{Xu}$ Chao \\ Tianyu Chen
}

Xiangjun Chen
Zheng Chunshan
Zhendong Cui
Feng Dai
Zhenxue Dai
Melvin Diaz
Janvier Domra Kana
Linming Dou
Feng Du
Claire Dusabemariya
Ashley Dyson
Walid El kamash
Lifeng Fan
Long Fan
Zhiqiang Fan
Gan Feng
Jinwei Fu
Shashika Gajanayake
Thomas Gallot
Ke Gao
Mingzhong Gao
Dmitry Garagash
Dr. Amitava Ghosh
Vyacheslav Glinskikh
Peilin Gong

\author{
Battista Grosso \\ Shib Group \\ Helong Gu \\ Hakan Güneyli Güneyli \\ Yanhui Han \\ Xianjie Hao \\ Mengbing $\mathrm{He}$ \\ Chen Hongran \\ Sakhawat Hossain \\ Zhengmeng Hou \\ Dawei Hu \\ Mengsu $\mathrm{Hu}$ \\ Haiying Huang \\ Na Huang \\ MD Aminul Islam \\ Panteleev Ivan \\ Nagham Jasim Al-Ameri \\ Yinlin Ji \\ Yunzhong Jia \\ Quan Jiang \\ Yu-Yong Jiao \\ Yang Ju \\ Ram Chandar Karra, \\ Majid Khan \\ Jahangir Khan
}


Manoj Khandelwal

Piu Kundu

Jin Lai

Bobo Li

Changdong Li

Diyuan Li

Guichen Li

Hua Li

Lianchong Li

Qi Li

Sanbai Li

Xiaofeng Li

Xingmail Li

Yingchun Li

Yong $\mathrm{Li}$

Ming Li

Yingchun Li

Haojie Lian

Zhiyi Liao

Lichuanqi Lichuanqi

Huasheng Lin

Peng Lin

Huihai liu

Kai Liu

Kouqi Liu

Liansheng Liu

Shao Liu

Xiaoli Liu

Yong Liu

Zhenghe Liu

Wasantha Liyanage

Diego Lo Presti

Renchao Lu

Shouqing Lu

Qiao Lyu

Haichun Ma

Yankun ma

Yifei Ma

Bankim Mahanta

Sukru Merey

Brijes Mishra

Rudrajit Mitra

Faizal Mohammed

Masoud Monjezi

Hem Bahadur Motra

Bassem Nabawy

Pengpeng Ni

Baisheng Nie

Peter Niemz

Fulong Ning
Zhongrong Niu

Hiroyuki Noda

Yildiray Palabiyik

Kang Peng

Indra Permanajati

Purwanto Purwanto

Jiadong Qiu

Hanumantha Rao

Goc Romain

Jonny Rutqvist

C.S. Sandeep

Souvik Sen

Kostas Senetakis

Eyal Shalev

Xueyi Shang

Zhushan Shao

Xiaomeng Shi

Benjian Song

Zhenlong Song

Qiang Sun

Fujiao Tang

Jiren Tang

Xuhai Tang

Ming Tao

Fei Tian

Manolis Veveakis

Vikram Vishal

Chunguang Wang

Dengke Wang

Fei Wang

Gang Wang

Huaning Wang

Jianguo Wang

Jiehao Wang

Jun Wang

Lei Wang

Peitao Wang

Qi Wang

Shanyong Wang

Shiming wang

Shugang Wang

Tingting Wang

Wensong Wang

Xian Yi Wang

Yunteng Wang

Zewei Wang

Zhihe Wang

Dinesha Wanigarathnajayasekara

Kumari Wanniarachchige

Pabasara Wanniarachchige
Fei Wu

Wei Wu

Xuezhen Wu

$\mathrm{Yu} \mathrm{Wu}$

Zhijun Wu

Kaiwen Xia

Songqiang Xiao

Jiang $\mathrm{Xu}$

Songlin $\mathrm{Xu}$

Tao Xu

Xiaomeng $\mathrm{Xu}$

Zhenhao Xu

Lei Xue

Diansen Yang

Mijia Yang

Sheng-Qi Yang

Weimin Yang

Xiaobin Yang

Wendong Yang

Chi Yao

Hideaki Yasuhara

Bin $\mathrm{Yu}$

Liyuan Yu

Miao Yu

Song Yu

Ioannis Zevgolis

Chuanqing Zhang

Dongming Zhang

Fengshou Zhang

Guanglei Zhang

Hongwei Zhang

Kai Zhang

Pengcheng Zhang

Ru Zhang

Shiwei Zhang

Yi Zhang

Yong Zhang

Zhenyu Zhang

Gaofeng Zhao

Kang Zhao

Wanchun Zhao

Zhihong Zhao

Zhiye Zhao

Fei Zheng

Yanlong Zheng

Changtai Zhou

Hui Zhou

Jian Zhou

Tao Zhou

Yingfang Zhou 


$\begin{array}{lll}\text { Zongqing Zhou } & \text { Jianbo Zhu } & \text { Li Zhuang } \\ \text { Cheng Zhu } & \text { Qizhi Zhu } & \text { Junpeng Zou } \\ \text { Chun Zhu } & \text { Zhen Zhu } & \text { Yu-jun Zuo }\end{array}$

We apologize for any errors or inadvertent omissions.

Publisher's Note Springer Nature remains neutral with regard to jurisdictional claims in published maps and institutional affiliations. 